\title{
Cistectomía radical como tratamiento del cáncer vesical infiltrante en el paciente de edad avanzada
}

\author{
March Villalba JA, Martínez Jabaloyas JM, Pastor Hernández F, Günthner Stefan FJ, \\ Rodríguez Navarro R, Chuan Nuez P.
}

Servicio de Urología del Hospital Clínico Universitario de Valencia. Valencia.

Actas Urol Esp. 2008;32(7):696-704

\section{RESUMEN}

CISTECTOMÍA RADICAL COMO TRATAMIENTO DEL CÁNCER VESICAL INFILTRANTE EN EL PACIENTE DE EDAD AVANZADA

Introducción: Realizar una cistectomía radical en ancianos con cáncer vesical infiltrante es un tema controvertido.

Objetivo: El objetivo de nuestro trabajo ha sido determinar si existen diferencias significativas en cuanto a la morbimortalidad perioperatoria, complicaciones tardías, tiempo libre de enfermedad y supervivencia cáncer-específica en un grupo de pacientes ancianos con cáncer vesical infiltrante tratados mediante cistectomía radical, comparado al resto de pacientes de menor edad y analizar qué variables pudieron predecir la morbi-mortalidad perioperatoria.

Material y métodos: Estudio retrospectivo de pacientes con cáncer vesical infiltrante tratados mediante cistectomía radical y derivación ileal. Se compararon 2 grupos: $<70$ años en el momento de la realización de la cistectomía (n=55) y $\geq 70$ años (n=57).

Resultados: No hubo diferencias entre ambos grupos con respecto a: tiempo quirúrgico, complicaciones intraoperatorias $(<70=21,8 \%, \geq 70=31,6 \%)$, mortalidad postoperatoria $(<70=3,6 \%, \geq 70=8,8 \%)$, complicaciones postoperatorias menores $(<70=18,2 \%, \geq 70=26,3 \%)$ y médicas $(<70=7,3 \%, \geq 70=8,8 \%)$, eventos tardíos, periodo libre de enfermedad y supervivencia cáncer-específica. El porcentaje de complicaciones mayores $(<70=23,6 \%, \geq 70=43,9 \%)$ y la estancia hospitalaria $(<70=10,2$, $\geq 70=15,2$ días) difieren significativamente entre ambos grupos. La edad y los factores de riesgo cardiacos predicen de manera independiente la aparición de complicaciones postoperatorias mayores.

Conclusiones: La cistectomía radical puede ser realizada en pacientes ancianos seleccionados adecuadamente.

Palabras clave: Anciano. Cistectomía. Cáncer vesical infiltrante.

\section{ABSTRACT}

RADICAL CYSTECTOMY AS A MUSCLE-INVASIVE BLADDER CANCER TREATMENT IN ELDERLY PATIENTS

Introduction: Radical cystectomy in elderly is a controversial issue that increases importance overtime because average life span is growing.

Objetive: The porpouse of our work was to analize the differences about perioperative and later outcomes between ages of patients with muscle-invasive bladder neoplasm treated with radical cystectomy.

Material and methods: We retrospectively reviewed the records of patients who underwent radical cystectomy for muscle-invasive bladder cancer. Two age groups were compared: $<70$ years old at time of cystectomy $(\mathrm{n}=55)$ and $\geq 70$ years $(\mathrm{n}=57)$.

Results: There was no difference between both age groups about: time of surgery, intraoperative complications $(<70=21,8 \%, \geq 70=31,6 \%)$, postoperative mortality $(<70=3,6 \%, \geq 70=8,8 \%)$, minor $(<70=18,2 \%, \geq 70=26,3 \%)$ and major medical postoperative complications $(<70=7,3 \%, \geq 70=8,8 \%)$, late outcomes as cancer-specific morby-mortality and actuarial overall survival stratified by patient age. The rate of major postoperative complications $(<70=23,6 \%, \geq 70=43,9 \%)$ as well as the mean length of hospital stay $(<70=10,2, \geq 70=15,2$ days $)$ differed significantly between the two age groups. Age and cardiovascular risk factors were independient predictive factors of mayor postoperative complications.

Conclusions: Radical cystectomy could be performed in carefully selected elderly patients. 
$\mathrm{L}^{\mathrm{a}}$ a edad es un factor de riesgo para desarrollar cáncer vesical. Una persona de 70 años presenta una probabilidad 3 veces mayor de padecer dicho cáncer con respecto a otra con edad comprendida entre los 55-59 años y 15 veces mayor que las que se encuentran en el intervalo de edad de 30-54 años. Por este motivo y por el envejecimiento progresivo de la población, está aumentando la importancia del manejo del cáncer vesical en el anciano ${ }^{1-4}$.

Diferentes estudios defienden la indicación de una cistectomía radical como tratamiento del cáncer vesical infiltrante también en los pacientes ancianos; en cambio, otros afirman que dicha cirugía radical genera una gran morbi-mortalidad en este grupo de edad, por lo que sería necesario realizar en dichos pacientes un tratamiento conservador ${ }^{5-10}$.

La edad, según algunos autores, no influye en el periodo libre de enfermedad ni en la supervivencia cáncer-específica, de hecho, series de trabajos realizados en autopsias sugieren que el cáncer vesical metastatiza independientemente de la edad del paciente. No obstante, otros trabajos como el de Clark o Nielsen afirman que el paciente anciano ( $\geq 70$ años) presenta peor supervivencia y un tiempo libre de enfermedad más corto y exponen que la causa puede ser debida a: un periodo más prolongado desde el diagnóstico hasta la cistectomía, estadios anatomopatológicos más avanzados en el anciano y una menor predisposición al tratamiento con quimioterapia adyuvante ${ }^{11,12}$.

El objetivo de nuestro trabajo es describir y valorar si el grupo de pacientes mayores de 70 años diagnosticados de cáncer vesical infiltrante y tratados mediante cistectomía radical por nuestro grupo, presenta diferencias en cuanto a la morbi-mortalidad perioperatoria y el seguimiento postoperatorio a largo plazo, con respecto al resto de pacientes de la serie. También se analiza si la edad avanzada, aparte de otras variables, influye en la morbi-mortalidad perioperatoria, en la recidiva clínica de la enfermedad y en la supervivencia cáncer-específica.

\section{MATERIAL Y MÉTODOS}

Estudio retrospectivo de 112 pacientes diagnosticados de cáncer vesical infiltrante tratados mediante cistectomía radical y derivación ileal tipo Bricker en un periodo de 7 años. Los criterios de exclusión fueron: presencia de enfermedad diseminada previa a la cistectomía y pacientes a los que se les ha realizado una cistectomía de rescate.
El examen clínico antes de la cirugía incluyó: anamnesis, exploración física, hemograma, bioquímica general, radiografía de tórax, electrocardiograma y una TC abdomino-pélvica para completar el estudio clínico de extensión.

La técnica quirúrgica realizada consistió en una cistoprostatectomía radical mediante laparotomía media hipogástrica y linfadenectomía ilio-obturatriz bilateral, optando por la reconstrucción de un conducto ileal tipo Bricker como derivación urinaria.

El seguimiento de los pacientes tras la cirugía consistió en una primera visita a los 15-30 días, seguida de una visita a los 3 meses y con posterioridad, una cada 6 meses durante 3 años, en la cual se solicitó analítica y TC de control. El paciente que no presentó recidiva o progresión de la enfermedad tras estos 3 años, fue seguido con una periodicidad anual.

En el análisis comparativo de la muestra, los pacientes se clasificaron dependiendo de la edad en el momento de la cistectomía en 2 grupos $(<70$ y $\geq 70$ años).

\section{Análisis del periodo perioperatorio}

Comprende los 30 días tras la intervención quirúrgica (periodo intraoperatorio inclusive) ${ }^{13,14}$

Las variables dicotómicas preoperatorias fueron: sexo, tabaco (fumador activo), alcohol (ingesta moderada/importante) y la existencia de co-morbilidad como factores de riesgo cardicacos, pulmonares, vasculares (macroangiopatía), hipertensión arterial, diabetes mellitus, cirugía mayor previa (torácica, retroperitoneal, abdominal o pélvica) y otros (hepatopatía, insuficiencia renal). También se clasificó a los pacientes según la puntuación preoperatoria ASA (American Society of Anesthesiologists) en 2 grupos (1-2/3-4) ${ }^{15}$.

Las variables postoperatorias recogidas fueron: tiempo quirúrgico (minutos), estancia en reanimación (días), unidades de hemoconcentrados transfundidos y días de estancia hospitalaria.

Se calcularon las tasas de mortalidad y de morbilidad tanto intraoperatoria como postoperatoria. La morbilidad también se clasificó según la gravedad en complicaciones mayores y menores, y según la etiología en complicaciones a causa de la cirugía y por causa médica, según las publicadas en otros estudios $^{6,7,13,15,16}$.

En el análisis descriptivo de la morbilidad, también se recogieron y los tipos de complicaciones sufridas $^{13}$. 
Para detectar qué variables de las anteriores (incluyendo la edad) predicen de manera independiente la morbi-mortalidad perioperatoria, se realizó un análisis de regresión logística multivariante, siendo las variables dependientes analizadas (no presencia/presencia de): mortalidad postoperatoria, morbilidad intraoperatoria, complicaciones mayores y menores.

También se muestran los resultados anatomopatológicos tras la cistectomía según los dos grupos de edad. Han sido estratificados según el estadio (pT2 (a y b), zpT3) y la presencia/ausencia de adenopatías $(\mathrm{pNO}, \mathrm{pN}+)$.

\section{Análisis de la evolución posterior}

Para analizar el periodo libre de enfermedad (recidiva clínica) y la supervivencia (mortalidad cáncerespecífica) se obtuvieron las gráficas de supervivencia actuarial (método de Kaplan Meier), estratificando la muestra en los dos grupos de edad analizados (punto de corte en 70 años) ${ }^{12,13}$, comparando las medianas de ambos periodos de seguimiento. Se ha considerado un límite de seguimiento mínimo de 6 meses.

El método estadístico utilizado en el análisis univariante de la muestra fue primero el test de normalidad para la variable edad y posteriormente, un análisis de contingencia Chi-cuadrado para las variables categóricas y el test t-student para las variables continuas. En la regresión logística multivariante se calculó la Odds ratio y su intervalo de confianza (95\%).

Se empleó el programa SPSS 12.0 como paquete estadístico informático, estableciendo el límite de significación estadística en el 5\% $(\mathrm{p}<0,05)$.

\section{RESULTADOS}

La muestra compuesta de 112 Factores de riesgo (FR) pacientes presenta una media de edad de 68,8 \pm 8,84 años, y una mediana de 70,2 $(39,9-86,1)$ años. La variable edad sigue una distribución normal.

La mediana de edad del grupo $<70$ años es de 63,5 (39,9-69,9) años y la del grupo $\geq 70$ años es de 75,5 (70,1-86,2). Un 56,1\% de los pacientes con edad $\geq 70$ años tienen más de 75 y un 15,8\% una edad mayor o igual a 80 años.

\section{Análisis de la morbi-mortalidad en el periodo perioperatorio}

En el grupo de pacientes con edad $\geq 70$ años existe mayor porcentaje de hipertensión, cardiopatía, neumopatía, antecedentes de cirugía mayor y de otros factores de riesgo como la insuficiencia renal o hepatopatía. En cambio, en este grupo hay menor número de fumadores, diabéticos y de pacientes que ingieren alcohol en cantidad moderada/importante. Con respecto a la puntuación ASA, en el grupo $\geq 70$ años hay mayor proporción de pacientes con ASA 34. Todas las diferencias que existen entre ambos grupos de edad con respecto a los factores de riesgo preoperatorios y la clasificación ASA, no llegan a alcanzar significación estadística (p>0,05), (Tabla 1).

En cuanto a las variables operatorias, el grupo de edad $\geq 70$ años ha presentado un tiempo quirúrgico medio de 247 minutos, similar al tiempo del grupo $<70$ años (235 minutos) (p>0,05).

La morbilidad intraoperatoria ha sido mayor en el grupo $\geq 70$ años $(31,6 \%)$ con respecto al otro grupo de menor edad $(21,8 \%)$, pero sin ser la diferencia entre ambos grupos significativa. La principal complicación intraoperatoria ha consistido en la necesidad de transfundir 2 o más unidades de hemoconcentrados durante la intervención. No se ha dado ningún caso de mortalidad intraoperatoria (Tabla 2).

Con respecto a la morbilidad postoperatoria, ambos grupos han permanecido un día de media en reanimación. Los pacientes ancianos han requerido
Tabla 1. Diferencias entre los grupos de edad respecto a los factores de riesgo preoperatorios

\begin{tabular}{lccc}
\hline & $<\mathbf{7 0}$ a. $(\mathbf{N}=\mathbf{5 5})$ & $\mathbf{2 7 0}$ a. $(\mathbf{N = 5 7 )}$ & Valor de $\mathbf{p}$ \\
\hline Media de Edad (años) & $61,8(39,9-69,9)$ & $75,6(70,2-86,2)$ & $\mathrm{p}<0,029$
\end{tabular}

Sexo: varón

Sexo: mujer

Fumador

Hipertensión arterial

Cirugía abdomino-pélvica

Diabetes mellitus

Cardiacos

Alcohol (moderado/severo)

Pulmonares

Vasculares

$$
\begin{gathered}
1(1,8 \%) \\
26(47,3 \%) \\
15(27,3 \%) \\
14(25,5 \%) \\
13(23,6 \%) \\
9(16,4 \%) \\
8(14,5 \%) \\
6(10,9 \%) \\
3(5,5 \%) \\
9(16,4 \%)
\end{gathered}
$$

$54(98,2 \%)$

Otros

\section{Puntuación ASA}

ASA 1-2

$29(52,7 \%)$

$26(47,3 \%)$

$49(86,0 \%)$
$8(14,0 \%)$
$13(22,8 \%)$
$24(42,1 \%)$
$20(35,1 \%)$
$8(14,0 \%)$
$17(29,8 \%)$
$3(5,3 \%)$
$11(19,3 \%)$
$5(8,8 \%)$
$17(29,8 \%)$

$22(38,6 \%)$
$35(61,4 \%)$

0,734

0,186

0,133

0,336

0,553

0,578

0,329

0,454

0,543

0,954

0,329

$35(61,4 \%)$

0,527

0,408
ASA 3-4 
un mayor número de unidades de hemoconcentrados que el grupo <70 años (media de 3,58 frente a 1,7 unidades) $(\mathrm{p}<0,05)$ y han presentado una estancia media hospitalaria más prolongada $(15,2$ días frente a 10,2) $(\mathrm{p}<0,05)$ (Tabla 2).

Los pacientes ancianos han sufrido en el periodo perioperatorio un mayor porcentaje de complicaciones mayores que el grupo $<70$ años $(43,9 \%$ frente a un 23,6\%) ( $<<0,05)$. Dentro de estas complicaciones cabe destacar que en el grupo de ancianos sólo predominan las sucedidas a causa de la cirugía con respecto al grupo de menor edad ya que ambos grupos han obtenido un porcentaje similar de complicaciones mayores de causa médica (Tabla 3).

Con respecto a las complicaciones mayores de causa quirúrgica, las más frecuentes en el grupo de ancianos fueron: la transfusión de $>2$ unidades de hemoconcentrados, la evisceración y el absceso intraabdominal. Las de causa médica más frecuentes fueron la hemorragia digestiva aguda y la trombosis venosa profunda/tromboembolismo pulmonar. Cabe destacar como complicación médica la presencia de desorientación en un 5,3\% de los pacientes ancianos, complicación que no ha ocurrido en los pacientes de menor edad (Tabla 3).

Respecto a las complicaciones de menor gravedad, son más frecuentes en el grupo de ancianos $(26,3 \%$ frente a un $18,2 \%$ ), pero sin llegar a alcanzar una significación estadística ( $p>0,05)$. De entre estas complicaciones, el íleo prolongado ocurre por igual en ambos grupos de edad, siendo la más frecuente la infección superficial de la herida y/o la aparición de seroma (Tabla 3).

En cuanto a la mortalidad postoperatoria, a pesar de que es algo más elevada en el grupo de ancianos $(8,8 \%$ frente a un $3,6 \%)$, dicha diferencia no es significativa $(\mathrm{p}>0,05)$ (Tabla 2).
Si analizamos todas las variables recogidas con el fin de averiguar cuáles pueden predecir de manera independiente la morbi-mortalidad perioperatoria, hemos encontrado que la edad ha sido factor de riesgo independiente en cuanto a las complicaciones mayores (OR: 4,64) ( $\mathrm{p}<0,05)$, pero no con respecto a la mortalidad postoperatoria, morbilidad intraoperatoria ni con las complicaciones menores (Tablas 4 y 5).

Tabla 2. Diferencias entre los grupos de edad respecto a las variables perioperatorias

\begin{tabular}{lccc}
\hline & $<\mathbf{7 0}$ a. $(\mathbf{N}=\mathbf{5 5})$ & $\mathbf{\geq 7 0}$ a. $(\mathbf{N = 5 7 )}$ & Valor de $\mathbf{p}$ \\
\hline Tiempo operatorio (min.) & $235(200-420)$ & $247(200-660)$ & 0,309 \\
Morbilidad Intraoperatoria* & $12(21,8 \%)$ & $18(31,6 \%)$ & 0,533 \\
Días en Reanimación & $1,04(0-3)$ & $1,11(0-6)$ & 0,695 \\
Hemoc.transfundidos & $1,7(0-9)$ & $3,58(0-12)$ & 0,008 \\
Estancia hospitalaria (días) & $10,2(6-61)$ & $15,2(7-44)$ & 0,006 \\
Mortalidad & & & \\
Postoperatoria* & $2(3,6 \%)$ & $5(8,8 \%)$ & 0,728 \\
Intraoperatoria & 0 & 0 & -
\end{tabular}

*Número de pacientes (proporción).

Tabla 3. Diferencias entre los grupos de edad respecto a los tipos de complicaciones perioperatorias (morbilidad) expresadas en número de eventos, excepto *en número de pacientes (proporción)

\begin{tabular}{|c|c|c|c|}
\hline & $<70$ a. $(\mathrm{N}=55)$ & $\geq 70$ a. $(N=57)$ & Valor de $p$ \\
\hline MAYORES* & $13(23,6 \%)$ & $25(43,9 \%)$ & 0,039 \\
\hline Causa quirúrgica* & $13(23,6 \%)$ & 25 (43,9\%) & 0,039 \\
\hline Transfusión de $>2$ hc & 11 & 14 & - \\
\hline Evisceración & 0 & 6 & - \\
\hline Absceso intraabdominal & 0 & 3 & - \\
\hline Lesión vásculo-nerviosa & 1 & 2 & - \\
\hline Dehiscencia s. Ileo-ileal & 0 & 2 & - \\
\hline Linforrea & 1 & 0 & - \\
\hline Peritonitis & 0 & 1 & - \\
\hline Causa médica* & $4(7,3 \%)$ & $5(8,8 \%)$ & 0,841 \\
\hline Infarto agudo miocardio & 0 & 1 & - \\
\hline Hemorragia digestiva & 1 & 2 & - \\
\hline TVP/TEP & 1 & 2 & - \\
\hline Neumonía/atelectasia & 0 & 1 & - \\
\hline Edema agudo de pulmón & 1 & 0 & - \\
\hline Sock séptico & 1 & 1 & - \\
\hline MENORES* & $10(18,2 \%)$ & $15(26,3 \%)$ & 0,608 \\
\hline Causa quirúrgica* & $10(18,2 \%)$ & $15(26,3 \%)$ & 0,608 \\
\hline Íleo prolongado & 4 & 4 & - \\
\hline Infección herida/seroma & 6 & 12 & - \\
\hline Causa médica* & 0 & $3(5,3 \%)$ & 0,601 \\
\hline Desorientación & 0 & 3 & - \\
\hline
\end{tabular}


Tabla 4. Análisis multivariante mediante regresión logística de los factores de riesgo con respecto a la morbilidad intraoperatoria y la mortalidad postoperatoria.

\begin{tabular}{lcccccc}
\hline & \multicolumn{3}{c}{ Mortalidad postoperatoria } & \multicolumn{3}{c}{ Morbilidad intraoperatoria } \\
\hline & $* *$ OR & $*$ (IC95\%) & Valor de $\mathbf{p}$ & $* *$ OR & *(IC95\%) & Valor de p \\
\hline Sexo (H/M) & 0,87 & $(0,05-15,68)$ & 0,92 & $\mathbf{7 , 9 5}$ & $(\mathbf{1 , 3 4 - 4 7 , 1 0 )}$ & $\mathbf{0 , 0 2}$ \\
Edad $(\geq 70 /<70)$ & 0,38 & $(0,10-2,48)$ & 0,31 & 1,24 & $(0,40-3,80)$ & 0,71 \\
Fumador & 0,72 & $(0,08-6,48)$ & 0,76 & 0,93 & $(0,29-3,0)$ & 0,90 \\
Alcohol & 0,00 & & - & 0,00 & & - \\
ASA (3-4 / 1-2) & 0,99 & $(0,10-9,41)$ & 0,99 & 1,92 & $(0,58-6,41)$ & 0,29 \\
Cir abdomin-pelv & 0,62 & $(0,08-4,79)$ & 0,64 & 0,79 & $(0,24-2,56)$ & 0,69 \\
Diabetes mellitus & 1,6 & $(0,00-2,00)$ & 0,99 & 1,44 & $(0,40-5,11)$ & 0,58 \\
HTA & 1,18 & $(0,16-8,53)$ & 0,86 & 0,66 & $(0,20-2,15)$ & 0,49 \\
FR cardiacos & 1,98 & $(0,18-21,99)$ & 0,57 & 1,48 & $(0,44-5,02)$ & 0,53 \\
FR pulmonares & 0,84 & $(0,06-11,74)$ & 0,89 & 2,15 & $(0,53-8,75)$ & 0,28 \\
FR Vasculares & 0,18 & $(0,01-4,45)$ & 0,29 & 0,52 & $(0,04-7,16)$ & 0,63 \\
Otros FR & 0,00 & & - & 0,57 & $(0,15-2,06)$ & 0,38 \\
\hline
\end{tabular}

**OR: Odds ratio. *IC95\%: Intervalo de confianza.

Tabla 5. Análisis multivariante mediante regresión logística de los factores de riesgo con respecto a las complicaciones menores y mayores

\begin{tabular}{|c|c|c|c|c|c|c|}
\hline & \multicolumn{3}{|c|}{ Morbilidad C. menores } & \multicolumn{3}{|c|}{ Morbilidad C. mayores } \\
\hline & OR & (IC95\%) & Valor de $\mathbf{p}$ & OR & (IC95\%) & Valor de $p$ \\
\hline Sexo $(H / M)$ & 0,47 & $(0,66-3,32)$ & 0,45 & 3,13 & $(0,27-3,70)$ & 0,37 \\
\hline Edad $(\geq 70 /<70)$ & 2,48 & $(0,84-7,34)$ & 0,10 & 4,64 & $(1,10-19,49)$ & 0,03 \\
\hline Fumador & 1,90 & $(0,59-6,08)$ & 0,28 & 0,98 & $(0,24-4,13)$ & 0,98 \\
\hline Alcohol & 1,37 & $(0,27-6,89)$ & 0,71 & 1,45 & $(0,20-10,60)$ & 0,72 \\
\hline ASA $(3-4 / 1-2)$ & 0,58 & $(0,16-2,03)$ & 0,39 & 0,78 & $(0,15-4,05)$ & 0,77 \\
\hline Cir abdomin-pelv & 2,05 & $(0,71-5,86)$ & 0,18 & 0,25 & $(0,05-1,21)$ & 0,09 \\
\hline Diabetes mellitus & 0,72 & $(0,18-2,90)$ & 0,64 & 0,21 & $(0,20-2,27)$ & 0,20 \\
\hline HTA & 1,68 & $(0,52-5,42)$ & 0,38 & 0,12 & $(0,18-0,78)$ & 0,27 \\
\hline FR cardíacos & 2,77 & $(0,79-9,76)$ & 0,11 & 6,34 & $(1,42-28,33)$ & 0,02 \\
\hline FR pulmonares & 0,90 & $(0,18-4,47)$ & 0,89 & 0,65 & $(0,05-8,55)$ & 0,75 \\
\hline FR Vasculares & 1,33 & $(0,15-11,39)$ & 0,79 & 2,45 & $(0,17-36,18)$ & 0,52 \\
\hline Otros FR & 0,51 & $(0,13-2,05)$ & 0,34 & 1,05 & $(0,18-6,34)$ & 0,95 \\
\hline
\end{tabular}

**OR: Odds ratio. *IC95\%: Intervalo de confianza.

Otras variables también independientes son: sexo (mujer) en cuanto a la morbilidad intraoperatoria $(\mathrm{OR}: 7,95)(\mathrm{p}<0,05)$ y la presencia de factores de riesgo cardiacos con respecto a sufrir complicaciones mayores (OR: 6,34) $(\mathrm{p}<0,05)($ Tablas 4 y 5).

A pesar de que existe un riesgo 1,96 veces superior de sufrir complicaciones intraoperatorias si el paciente presenta ASA 3-4, la puntuación ASA no ha obtenido significación en nuestro análisis ( $>0$,05) (Tablas 4 y 5).
Con respecto a los resultados anatomopatológicos, no se han observado diferencias entre los dos grupos de edad $(p>0,05)$. En cuanto a la extensión, la mayor parte de tumores vesicales invadían grasa perivesical (pT3), ocurriendo en ambos grupos de edad. Los pacientes $\geq 70$ años han presentado un mayor porcentaje de afectación linfática, pero éste no ha llegado a ser significativo con respecto al grupo $<70$ años $(p<0,05)$ (Tabla 6). 


\section{Seguimiento posterior de los pacientes tras el periodo perioperatorio.}

El periodo medio de seguimiento de los pacientes ha sido de $27,9 \pm 24,12$ meses $(1,12-93,14)$.

La causa más frecuente de morbilidad en los $<70$ años ha sido la presencia de estenosis en la anatomosis urétero-ileal asociada a insuficiencia renal obstructiva seguida de pielonefritis y eventración. En los pacientes con edad $\geq 70$ años ha sido la presencia de îleo obstructivo, fístula îleo-cutánea y eventración (Tabla 7).

La diferencia entre la tasa de reintervención quirúrgica como consecuencia de la cistectomía y/o la derivación tipo Bricker (16,6\% en el grupo $<70$ años frente a un $19 \%$ de los $\geq 70$ años) no ha sido significativa $(p>0,05)$ (Tabla 7$)$.

Con respecto a la recidiva de la enfermedad neoplásica, aunque el grupo <70 años obtiene una mediana del periodo libre de enfermedad mayor que los pacientes con edad $\geq 70$ años $(64,2$ frente a 43,5 meses), dicha diferencia no es significativa $(p>0,05)$. Ocurre lo mismo en cuanto a la supervivencia/mortalidad cáncer-específica, obteniendo una mediana de supervivencia en el grupo $<70$ años de $86,9(24,81-149,12)$ meses frente a la del grupo $\geq 70$ años, 64,3 (15,05 -133,15) meses (p>0,05) (Figs. 1 y 2).

Tabla 6. Resultados anatomopatológicos tras la cistectomía

\begin{tabular}{lccc}
\hline & $<\mathbf{7 0}$ a. $(\mathbf{N = 5 5 )}$ & $\mathbf{2 7 0}$ a. $(\mathbf{N = 5 7 )}$ & Valor de $\mathbf{p}$ \\
\hline p $\leq$ T2 pN0 & $15(27,27 \%)$ & $13(22,81 \%)$ & 0,87 \\
p T2 pN+ & $1(1,80 \%)$ & $1(1,74 \%)$ & 0,93 \\
$\geq$ pT3 pN0 & $21(38,20 \%)$ & $20(35,10 \%)$ & 0,90 \\
$\geq$ pT3 pN+ & $18(32,73 \%)$ & $23(40,35 \%)$ & 0,86 \\
\hline Bordes quirúrgicos afectos & $12(21,82 \%)$ & $11(19,30 \%)$ & 0,71
\end{tabular}

Tabla 7. Diferencias entre los grupos de edad respecto a las complicaciones tardías en el intervalo de seguimiento.

\begin{tabular}{lccc}
\hline Complicaciones tardías & $<\mathbf{7 0}$ a. (N=48) & $\mathbf{2 7 0}$ a. (N=42) & Valor de p \\
\hline Reintervención* & $8(16 \%)$ & $9(18 \%)$ & 0,59 \\
TVP/TEP & 1 & 0 & - \\
Insuf. renal obstructiva & 2 & 1 & - \\
Estenosis uretero-ileal & 3 & 1 & - \\
Eventración & 2 & 2 & - \\
Fístula ileo-cutánea & 1 & 2 & - \\
Parálisis ciático-poplíteo ext. & 1 & 1 & - \\
Hematuria & 1 & 1 & - \\
Pielonefritis & 2 & 1 & - \\
Íleo obstructivo & 1 & 2 & -
\end{tabular}

Reintervención expresada en $\mathrm{N}^{\circ}$ pacientes (proporción), el resto expresados según el número de eventos. *A causa de la cistectomía.
En resumen, con respecto al periodo postoperatorio, los pacientes ancianos presentan un mayor número de unidades de hemoconcentrados transfundidos, mayor estancia hospitalaria y un mayor porcentaje de complicaciones mayores a expensas de las de etiología quirúrgica.

La edad es un factor de riesgo independiente para desarrollar complicaciones mayores, como lo es también la presencia de factores de riesgo cardíacos.

Los pacientes ancianos no presentan diferencias en cuanto al porcentaje de complicaciones mayores postoperatorias de causa médica y de eventos tardíos como la tasa de morbi-mortalidad, periodo libre de enfermedad y supervivencia cáncer-específica con respecto al resto de pacientes.

\section{DISCUSIÓN}

Nuestro estudio compara el grupo de ancianos con el resto de edades, abarcando el periodo perioperatorio y el seguimiento posterior, incluyendo en éste último la diferencia entre los grupos en cuanto al tiempo de recidiva oncológica y a la supervivencia cáncer-específica. Este trabajo analiza la morbilidad tanto desde el punto de vista cuantitativo (tasas), como desde el punto de vista cualitativo (tipos de complicaciones mayores/menores y médicas/quirúrgicas recogidos como eventos). Respecto a la edad, en la actualidad es difícil establecer cuándo comienza la ancianidad, ya que el uso de criterios cronológicos, socio-culturales o biológicos pueden diferir en la edad de partida de dicho periodo. La tendencia de la mayoría de los trabajos científicos actuales es considerar como anciano al sujeto que ha superado los 70-75 años 6,13

La gerontología, distinguiendo entre vejez normal y patológica, subdivide los últimos periodos de la vida en: presenescencia, que abarca desde los 60 hasta los 70 años, vejez activa, desde los 70 hasta los 85 y senilidad cuando la edad supera los 85 . Por estos motivos hemos decidido que el punto de corte en este estudio sean los 70 años ${ }^{17,18}$. 


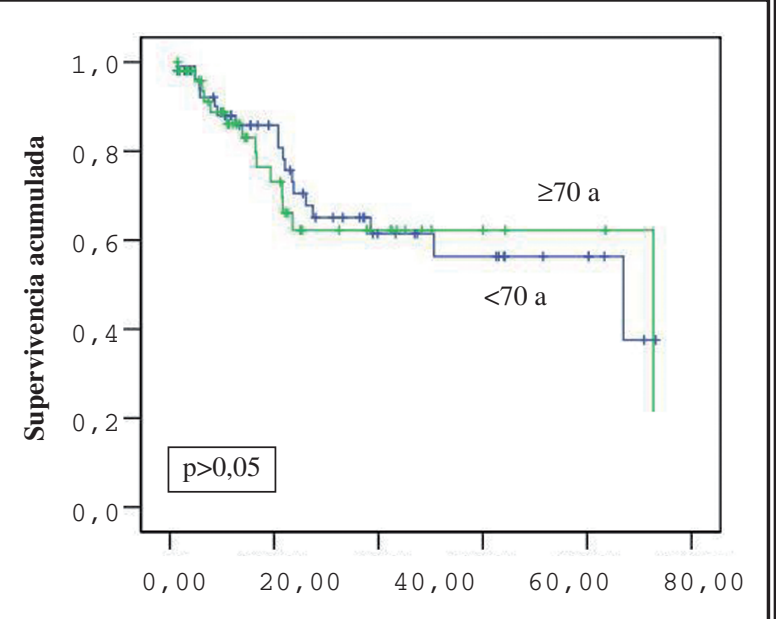

Tiempo libre de recidiva (meses)

Estimación según el método Kaplan-Meier del periodo libre de recidiva. Medianas, $<70$ años: $64,2(24,25-104,22)$ y $\geq 70$ años: 43,5 $(22,93-71,59)(\mathrm{p}>0,05)$

Figura 1. Periodo sin recidiva de enfermedad.

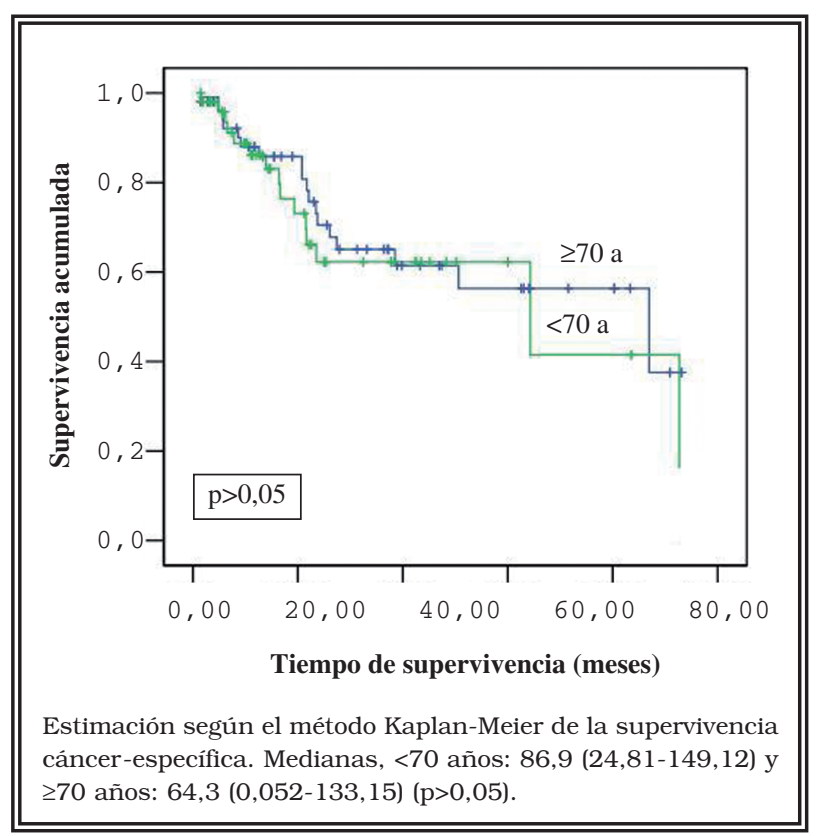

Figura 2. Supervivencia cáncer-específica.

La distribución normal y la diferencia de medias entre los grupos de edad de los pacientes de la muestra descarta sesgos de selección como, por ejemplo, tener muchos pacientes próximos a la edad de corte dentro de ambos grupos de edad.

El objetivo de realizar un análisis descriptivo de los factores de riesgo previos a la cirugía ha sido valorar a partir de qué tipo de muestra partimos. Demostrando que los pacientes ancianos presentan mayor co-morbilidad que los de menor edad, como se observa en la literatura científica y en la práctica clínica. El hecho de que las diferencias entre los subgrupos de edad lleguen, en la mayor parte de los factores de riesgo analizados, a no alcanzar la significación estadística en el análisis de contingencia, podría ser debido a la cantidad de la muestra.

En cuanto a los resultados perioperatorios de nuestro estudio: tasas de morbi-mortalidad, porcentaje de complicaciones, tiempo quirúrgico, estancia hospitalaria se encuentran dentro del rango de las publicadas por otros grupos $6,13,19,20$.

Los grados 3-4 de la clasificación ASA, aunque presentan una Odds ratio $>1$ en cuanto a la morbilidad intraoperatoria, en nuestra muestra no aporta valor predictivo significativo en cuanto a la morbi-mortalidad. En nuestro estudio ha sido el Servicio de Anestesiología el que ha clasificado a los pacientes, sin nuestra intervención, en cambio en los trabajos en los que se defiende su utilidad como predictor de la morbimortalidad postoperatoria tras la cistectomía en los ancianos, o no queda del todo claro quién es el que clasifica a los pacientes en las distintas puntuaciones ASA o son los propios investigadores los que lo realizan, produciéndose un sesgo de selección. Incluso algunos autores afirman que existe una tendencia a clasificar al anciano en grados ASA mayores, a pesar de que la edad no es un criterio de puntuación en esta escala y que los pacientes con ASA 2 presentan la misma morbi-mortalidad postoperatoria que los ASA 3, no siendo capaz de discriminar entre ellos ${ }^{15,21-23}$.

Según nuestro trabajo, las mujeres presentan un riesgo elevado de sufrir complicaciones intraoperatorias respecto al varón $(\mathrm{OR}=7,95 \mathrm{p}<0,05)$, no obstante no existe un mayor predominio de mujeres en el grupo de pacientes ancianos y el sexo no es un factor de riesgo independiente frente a la morbimortalidad postoperatoria. Estos hechos coinciden con el trabajo de Lee et $\mathrm{al}^{24}$.

La edad, en nuestro estudio, no influye en el periodo libre de enfermedad, ni en la supervivencia cáncer-específica, hecho que coincide con algunos trabajos $^{6,25}$. Los pacientes con edad menor de $<70$ años presentan mayor tasa de recurrencia y similar tiempo de recidiva que los ancianos.

En cuanto a la supervivencia cáncer-específica, se ha obtenido una tasa similar en ambos grupos y aunque los pacientes ancianos han presentado menor tiempo de supervivencia, este hecho no es significativo. 
En los trabajos que difieren con nuestros resultados, existen ciertas características a comentar. Nielsen et al. sólo encuentra diferencias significativas en cuanto a la recurrencia y la supervivencia cáncer específica en el grupo de pacientes con más de 80 años (resultados postoperatorios). No obstante, puede que influya en nuestros resultados el menor número de pacientes que posee la muestra ${ }^{11,12}$.

Nuestro estudio muestra que la cistectomía radical en un paciente anciano es un tratamiento con elevado riesgo de complicaciones durante el periodo perioperatorio ya que éstos presentan tasas más elevadas de complicaciones mayores, así como una mayor necesidad de hemoconcentrados y una estancia hospitalaria más prolongada que el grupo de menor edad. Sin embargo no podemos desestimar su realización en un paciente de edad avanzada porque, desde el punto de vista de nuestro trabajo, dichos pacientes no presentan diferencias significativas en el periodo perioperatorio en cuanto a la mortalidad y a las complicaciones de causa médica con respecto al resto de pacientes. Tampoco se han dado casos de mortalidad intraoperatoria.

En nuestro trabajo no se han presentado diferencias significativas entre ambos grupos de edad en cuanto al estadio pTN tras la cistectomía.

Una vez que el paciente anciano de nuestro estudio ha pasado el periodo post-operatorio, tampoco presenta diferencias con respecto a los pacientes de menor edad en cuanto a las complicaciones tardías, periodo libre de enfermedad y supervivencia cáncerespecífica. En nuestro trabajo, el grupo de pacientes $<70$ años presenta una mayor mediana de tiempo libre sin recidiva de la enfermedad y de supervivencia con respecto a los ancianos, pero estas diferencias no son significativas.

Por otra parte nosotros pensamos que el mal pronóstico de la enfermedad, la tórpida evolución y la falta de otras alternativas terapéuticas eficaces, llevan también a la elección de la cistectomía radical como la opción terapéutica en pacientes de edad avanzada.

Por este motivo, es necesario realizar una selección cuidadosa de los pacientes ancianos candidatos a la cistectomía radical con el fin de disminuir la morbilidad perioperatoria, utilizando criterios de exclusión como: la co-morbilidad presente y la situación clínica general en el momento de la cirugía (en nuestro estudio, los factores de riesgo cardiacos están asociados a una elevada tasa de complicaciones mayores), el nivel de dependencia y cognoscitivo del paciente anciano, pudiendo utilizar escalas métricas para ayudarnos a dicha valoración (Mini-mental, Karfnoski, etc.) ${ }^{21,26}$.

\section{CONCLUSIONES}

La cistectomía radical puede ser realizada en el paciente anciano, para ello es necesaria una selección cuidadosa de dichos pacientes, basándose en la morbilidad previa a la cirugía, en la situación clínica y en el nivel cognoscitivo y de dependencia de dicho paciente anciano.

\section{REFERENCIAS}

1. Anuario estadístico de España 1998-2005. Instituto Nacional de Estadística. Ministerio de Economía y Hacienda. www.ine.es (fecha de consulta 20/08/2007). Disponible en http://www.ine.es/ prodyser/pubweb/espcif/pobl07.pdf.

2. López-Abente G, Pollán M, Aragonés N, Pérez Gómez B, Hernández Barrera V, Lope V, et al. Situación del cáncer en España: Incidencia. An Sist Sanit Navar. 2004;27(2):165-173.

3. Otero Mauricio G., Fernández A., Gil Fabra J. Epidemiología descriptiva y analítica en Urología, oncológica. Tema monográfico del LXIII Congreso Nacional de Urología. Asociación Española de Urología. Jun 1998.

4. Pashos CL, Botteman MF, Laskin BL, Redaelli A. Bladder cancer. Epidemiology, diagnosis and management. Cancer pract. 2002;10(6):311-322.

5. Zebic N, Weinknecht S, Kroepfl D. Radical cystectomy in patients aged $>75$ years: an updated review of patients treated with curative and palliative intent. BJU int. 2005;95(9):12111214

6. Peyromaure M, Guerin F, Debre B, Zerbib M. Surgical management of infiltrating bladder cancer in elderly patients. Eur Urol. 2004;45(2):147-153.

7. Basso U, Bassi P, Sava T, Monfardini S. Management of muscle-invasive bladder cancer in the elderly. Expert Rev Anticancer Ther. 2004;4(6):1017-1035

8. George L, Bladou F, Bardou VJ, Gravis G, Tallet A, Alzieu C, et al. Clinical outcome in patients with locally advanced bladder carcinoma treated with conservative multimodality therapy. Urology. 2004;64(3):488-493.

9. Arias F, Dominguez MA, Martinez E, Illarramendi JJ, Miquelez S, Pascual I, et al. Chemoradiotherapy for muscle invading bladder carcinoma. Final report of a single institutional organ-sparing program. Int J Radiat Oncol Biol Phys.2000;47(2):373-378.

10. Danesi DT, Arcangeli G, Cruciani E, Altavista P, Mecozzi A, Saracino B, et al. Conservative treatment of invasive bladder carcinoma by transurethral resection, protracted intravenous infusion chemmotherapy, and hyperfractionated radiotherapy: long term results. Cancer. 2004;101(11):2540-2548.

11. Clark PE, Stein JP, Groshen SG, Cai J, Miranda G, Lieskovsky $\mathrm{G}$, et al. Radical cystectomy in the elderly. Comparison of survival between younger and older patients. Cancer. 2005;103(3): 546-552.

12. Nielsen ME, Shariat SF, Karakiewicz PI, Lotan Y, Rogers CG, Amiel GE, et al. Advanced age is associated with poorer bladder cancer-specific survival in patients treated with radical cystectomy. Eur Urol. 2007;51(3):699-706; discussion 706-708. 
13. Clark PE, Stein JP, Groshen SG, Cai J, Miranda G, Lieskovsky $\mathrm{G}$, et al. Radical cystectomy in the elderly. Comparison of clinical outcomes between younger and older patients. Cancer. 2005;104(1):36-43.

14. Chang S, Cookson MS, Baumgartner RG, Wells N, Smith JA Jr. Analysis of early complications after radical cystectomy:Results of a collaborative care pathway. J Urol. 2002;167(5):2012-2016.

15. Chang S, Alberts G, Cookson MS, Smith JA Jr. Radical cystectomy is safe in elderly patients at high risk. J Urol. 2001;166 (3):938-941.

16. Hollenbeck BK, Miller DC, Taub D, Dunn RL, Khuri SF, Henderson WG, et al. Identifying risk factors for potentially avoidable complications following radical cystectomy. J Urol. 2005; 174(4 Pt 1):1231-1237; discussion 1237.

17. Brenner H, Arndt V. Epidemiology in aging research. Exp.Geront. 2004;(39):679-686.

18. Bixquert Jimenez M, Gil Lita R. Gastroenterología geriátrica: Introducción. Bixquert Jimenez M, Gil Lita R. Gastroenterología geriátrica. Valencia, Ed Edide.2004, pag. 3-6.

19. Soulié M, Straub M, Gamé X, Seguin P, De Petriconi R, Plante $\mathrm{P}$, et al. A multicenter study of the morbidity of radical cystectomy in select elderly patients with bladder cancer. J Urol 2002;167(3):1325-1328.

20. Chang S, Baumgartner RG, Wells N, Cookson MS, Smith JA Jr. Causes of increased hospital stay after radical cystectomy in a clinical pathway setting. J Urol. 2002;167(1):208-211.

21. Editorial.The surgical risk of elderly patients with cancer. 2004 Surgical Oncology; 13:169-173.
22. Prout GR, Wesley MN, Yancik R, Ries LA, Havlik RJ, Edwards $\mathrm{BK}$, et al. Age and comorbidity impact surgical therapy in older bladder carcinoma patients. A population study. Cancer. 2005;104(8): 1638-1647.

23. Konety BR, Joslyn SA. Factors influencing aggresive therapy for bladder cancer:an analysis of data from the SEER program. 2003. J. Urol; 170:1765-1771.

24. Lee KL, Freiha F, Presti JC and Gill HS. Gender diferences in radical cystectomy: complications and blood loss. Urology. 2004;63(6): 1095-1099.

25. Figueroa AJ, Stein JP, Dickinson M, Skinner EC, Thangathurai D, Mikhail MS, et al. Radical cystectomy for elderly patients with bladder carcinoma. An updated experience with 404 patients. Cancer. 1997;83(1):141-147.

26. Weizer AZ, Joshi D, Daignault S, Kinnaman M, Hussain M, Montie JE, et al. Performance status is a predictor of overall survival of elderly patients with muscle invasive bladder cancer. J Urol. 2007;177(4):1287-1293.

Correspondencia autor: Dr. J.A. March Villalba Servicio de Urología. Hospital Clínico Universitario de Valencia Avda. Blasco Ibáñez, 17 - 46010 Valencia Tel.: 963862600

E-mail autor: joseantoniomarch@hotmail.com Información del artículo: Original - Cáncer vesical Trabajo recibido: septiembre 2007

Trabajo aceptado: febrero 2008 